\title{
UTILIDAD DE LA RESONANCIA MAGNETICA CON ENDOCOIL EN LA ETAPIFICACION Y PLANEAMIENTO DEL TRATAMIENTO DEL CANCER RECTAL
}

Dres. Manuel Fernández $A^{(1)}$, Hernán Aldana $V^{(1,2)}$, Jorge Larach $\mathbf{S}^{(3)}$, Andrés Larach $K^{(3)}$.

1. Departamento de Radiología, Clínica Las Condes. Chile.

2. Departamento de Radiología, Hospital Clínico Universidad de Chile.

3. Departamento de Cirugía, Clínica Las Condes. Chile.

\begin{abstract}
The rectal cancer has a different therapeutic approach due to its special anatomical location. Therefore, the surgical technique today it has been standardized and implay the total resection of the mesorectum to remove not just the rectal wall and the cancer but also all the fat around it and then the surgical plane is given by the MRF. To apply this technique and to know the use of co adjuvant pre surgical treatment the role of the MRI is essential because it is necessary to the exact extension of the tumor between the mesorectum and beyond the MRF. This excellence in the imaging quality it has been achieved with the use of the endorectal coil, which allows defining the all mesorectum, including the MRF, which will give the surgical plane, the preservation of the anal sphincter and the need of pre surgical treatment. From the information given by MRI the survival and recurrence rate of the rectal cancer, could be established.
\end{abstract}

Keywords: Endocoil, Magnetic resonance, Rectal cancer, Staging.

Resumen: El cáncer de recto tiene un enfoque terapéutico distinto al cáncer de colon, derivado de su ubicación anatómica. Esto ha llevado a estandarizar técnicas quirúrgicas específicas y en muchos casos a usar tratamiento coadyuvante previo, lo que requiere de una adecuada etapificación imaginológica que, si bien es igual a la clásica etapificación TNM, tiene algunos aspectos que son distintos y de extraordinaria importancia tanto para el planeamiento quirúrgico como para definir el tratamiento coadyuvante previo. La resonancia magnética (RM) juega un rol muy relevante, ya que debe definir la técnica

Fernández $\mathbf{M}$, y cols. Utilidad de la resonancia magnética con bobina endorrectal en la etapificación y planemiento del tratamiento del cáncer rectal. Rev Chil Radiol 2007; 13: $57-62$.

Correspondencia: Dr. Manuel Fernández A. manuel.fernandez@vtr.net quirúrgica, por ejemplo con o sin conservación del aparato esfinteriano, y la necesidad de tratamiento coadyuvante previo a la cirugía. La introducción de la RM de alta resolución, usando bobina endorrectal ha permitido obtener imágenes de óptima calidad, colocándola como la técnica de elección en el estudio de estos pacientes, sin negar la utilidad de la endosonografía en las lesiones superficiales.

Palabras clave: Cáncer rectal, Etapificación, Endocoil.

\section{Introducción}

El adenocarcinoma de recto, con una incidencia de 40 por 100.000 habitantes, es una causa común de muerte en Europa y Estados Unidos ${ }^{(1)}$. En nuestro país su incidencia ha aumentado sostenidamente en los últimos años alcanzando a 7.5 por 100.000 habitantes ${ }^{(2)}$. Tiene predilección por el sexo masculino, en especial mayor de 50 años.

El tratamiento de elección es quirúrgico. Actualmente, la técnica estandarizada es la resección total del mesorrectal (TME) o también llamada resección mesorrectal circunferencial $(\mathrm{RCM})^{(3)}$, es decir, la extirpación en bloque del recto con la grasa que le rodea, incluidas las estructuras linfáticas y vasculares. El plano de sección está dado por la fascia mesorrectal (FMR).

El pronóstico de la enfermedad está directamente relacionado al grado de extensión del tumor a través de la pared rectal hacia el mesorrecto y la capacidad de lograr un margen adecuado de resección circunferencial (RMC). Si el margen de resección es positivo, la recurrencia puede alcanzar hasta un $83 \%$, lo cual tiene un gran impacto en la calidad de sobrevida del paciente aun cuando el tiempo de sobrevida pudiera no variar significativamente ${ }^{(4)}$.

\section{Los objetivos de la RM son dos:}

a. Etapificación: es el objetivo general de las técnicas imaginológicas en la evaluación de las 
neoplasias. El desarrollo de la RM usando una combinación de bobina endorrectal y de superficie la han posicionado como la técnica de elección para la evaluación del compromiso en profundidad y ganglionar del cáncer rectal, factores que finalmente son determinantes del pronóstico.

b. Mapeo quirúrgico: definir la extensión del tumor en relación a la FMR, estructuras vecinas y su relación con el esfínter anal ya que la técnica quirúrgica y el pronóstico estarán dados no sólo por la clasificación TNM, sino también por la extensión del tumor y las posibilidades técnicas de realizar una cirugía con un amplio margen de sección y de preservación del aparato esfinteriano. Es así como los tumores T3, es decir con compromiso del mesorrecto, han sido subdivididos en tres subgrupos en base a la extensión del tumor dentro del mesorrecto, a lo cual ha contribuido enormemente la RM: a) aquéllos con un amplio margen de sección, b) con un escaso margen de sección y c) aquéllos con compromiso de la FMR. Esta subdivisión está basada en el diferente pronóstico de cada uno de estos subgrupos (y por lo tanto los resguardos quirúrgicos que deben tomarse). El último, es decir, con compromiso de la FMR, es comparable al tumor en etapa T4.

\section{Protocolos de Imagen}

La adecuada limpieza rectal, previa a la obtención de imágenes por RM, es fundamental para evitar errores de interpretación debido a artefactos derivados de residuos fecales.

En general, la distensión rectal se considera importante en la evaluación de las capas del recto, sin embargo se ha demostrado que el uso de cortes finos de alta resolución permite óptimos resultados sin necesidad de distender el recto ${ }^{(5)}$.

Las secuencias básicas del estudio han sido estandarizadas, existiendo dos corrientes: a) aquella que propone el uso de secuencias T2 solamente, técnica empleada por nosotros y b) el uso de secuencias T1 y T2. Estas adquisiciones son obtenidas en planos axial, coronal y sagital utilizando secuencias Turbo Spin Eco con grosor de corte de 3-5 $\mathrm{mm}$. y pequeño campo de visión (240-250 mm). Las secuencias con saturación grasa también han sido utilizadas para mejorar la visualización de la invasión tumoral en la grasa perirrectal. El uso de gadolinio (Gd) es también una contribución en la mayoría de los casos.

La introducción reciente de la RM con el uso combinado de bobinas de superficie (phased array) y endorrectales, ha permitido lograr imágenes de alta resolución con una detallada evaluación de la pared rectal, resolviendo el problema de la amplitud del área estudiada por la endosonografía, demostrando en forma similar al ultrasonido las distintas capas de la pared rectal ${ }^{(6)} \mathrm{y}$, gradualmente ha desplazado a la tomografía computada (TC) como herramienta de etapificación del cáncer rectal. La alta resolución de la RM sumada al alto contraste tisular de esta técnica, puede además mostrar el mesorrecto y estructuras vecinas. Su amplio campo de visión permite etapificar en forma óptima tumores avanzados con extensión al mesorrecto y estructuras vecinas.

En un número no despreciable de casos (20\%) el compromiso tumoral alcanza estructuras pelvianas vecinas, incluyendo la FMR, por lo que la RM contribuye a la selección de pacientes que requieren de radio y/o quimioterapia previa y también a la adecuada planificación del procedimiento quirúrgico.

\section{Correlación de imagen en RM y hallazgos pato- lógicos}

La identificación del grado de invasión neoplásica de la pared rectal se basa en la existencia de diferencias en la intensidad de señal en T2, donde la capa muscular (músculo liso) es notoriamente hipointensa y, con Gd marcadamente hiperintensa respecto del tumor que es sólo moderadamente hiperintenso tanto en T2 como en T1FS-Gd. La grasa perirrectal es marcadamente hiperintensa en T2, lo que permite diferenciar muy bien los ganglios que en esta secuencia son hipointensos. La FMR es visualizada como una fina línea hiperintensa en T2.

Los tumores en estadio T1 (Figura 1a,b,c), con invasión de mucosa y submucosa pero sin invasión de la muscular, son bien visualizados con bobina endorectal dada su gran resolución espacial, obteniendo resultados similares a la endosonografía.

Las neoplasias en etapa T2 están caracterizadas por la invasión de la capa muscular, la cual aparece engrosada en T2 y relativamente hipointensa con Gd en relación a las zonas no comprometidas (Figura 2 a,b). En la diferenciación entre T2 y T3 es crucial el compromiso de la grasa perirrectal.

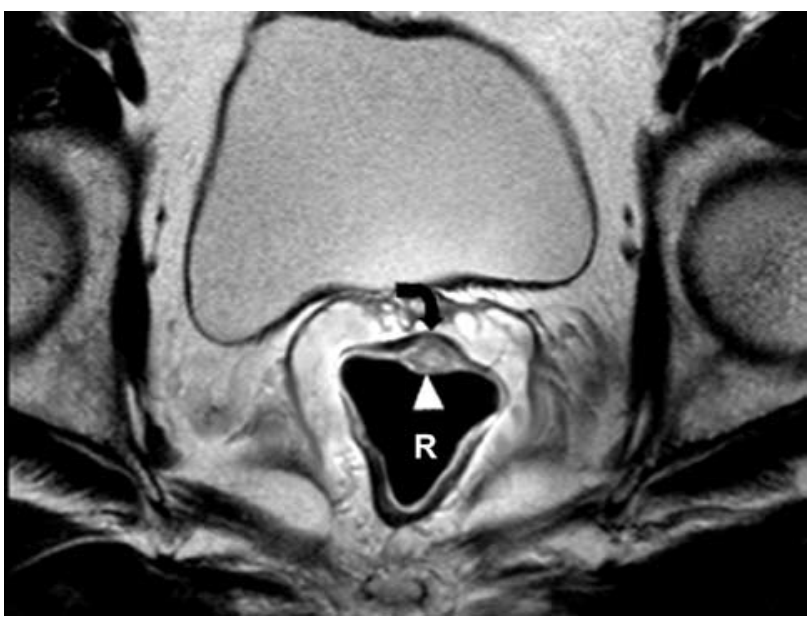

Figura 1a. T2, lesión elevada (cabeza de flecha) que compromete mucosa y submucosa, respetando la muscular (flecha curva) R: lumen rectal. 


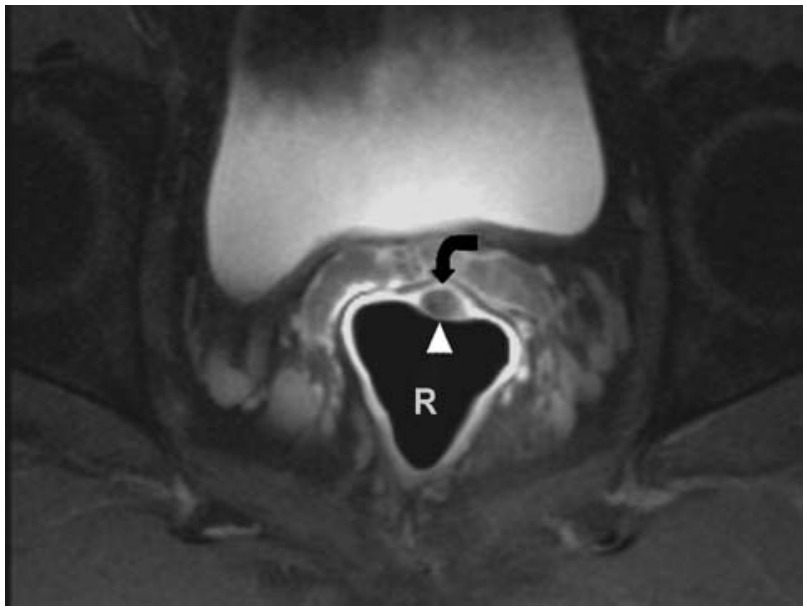

Figura 1b. La neoplasia (cabeza de flecha) se diferencia claramente de la muscular, que se ha hecho marcadamente hiperintensa (flecha curva) con el contraste, $\mathrm{Gd}$.

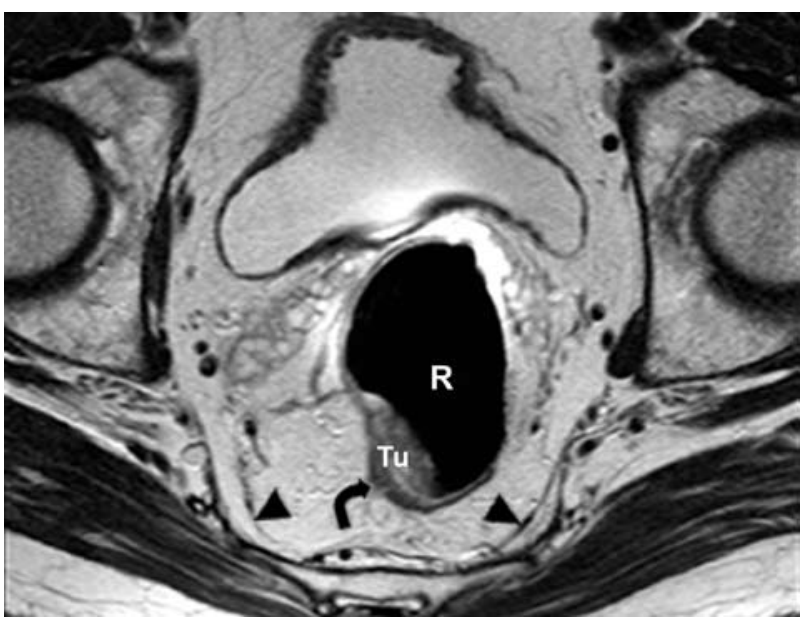

Figura 2 a. Neoplasia (Tu) que compromete la capa muscular (flecha curva) pero no invade la grasa mesorrectal. La FMR (cabezas de flechas) aparece demarcando nítidamente el límite del mesorrecto.

Las neoplasias T3 invaden la grasa del mesorrecto y esto es fácilmente visualizado con RM dada la diferencia de señal existente entre la grasa, que es hiperintensa en T2, y la baja señal del tejido tumoral. Dado que las secuencias usadas (T1FS) incluyen la saturación grasa (supresión de señal), cuando se usa Gd (Figura 3 a,b), la muscular resalta considerablemente e igualmente el tumor que aparece de mayor señal en el mesorrecto, donde la señal de la grasa ha sido suprimida (Figura $3 a, b$ ). En muchos casos probablemente se realiza sobre etapificación de T2 en T3, debido a la dificultad para diferenciar la espiculación desmoplásica en la grasa perirrectal causada sólo por fibrosis, es decir una etapa T2, de aquélla causada por invasión tumoral propiamente tal que corresponde a etapa $\mathrm{T} 3^{(7)}$. Sin embargo, este hecho no tiene mayor relevancia pues los estadios T2 y T3 requerirán de la misma técnica quirúrgica, RTM.

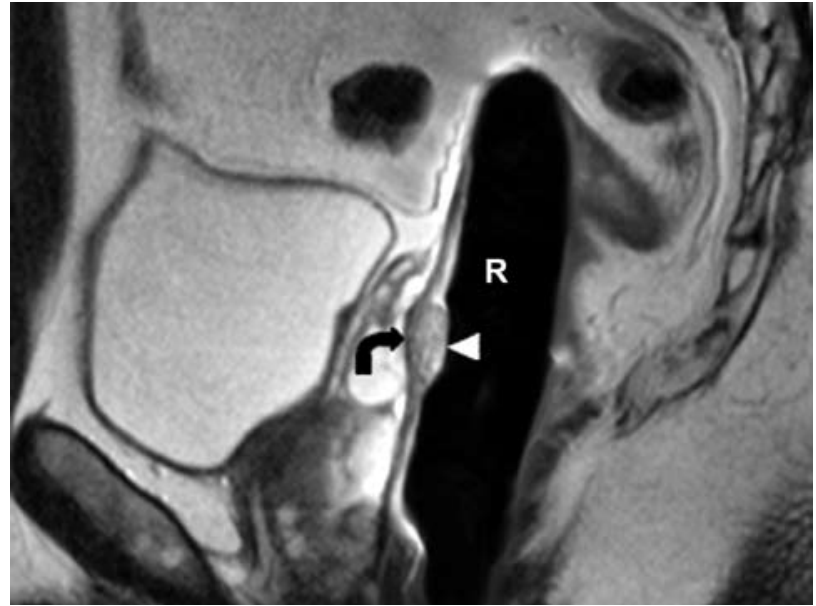

Figura 1c. T2 sagital. Corte muy útil para definir la distancia del tumor respecto de estructuras anatómicas como el esfínter, elevador del ano, etc.

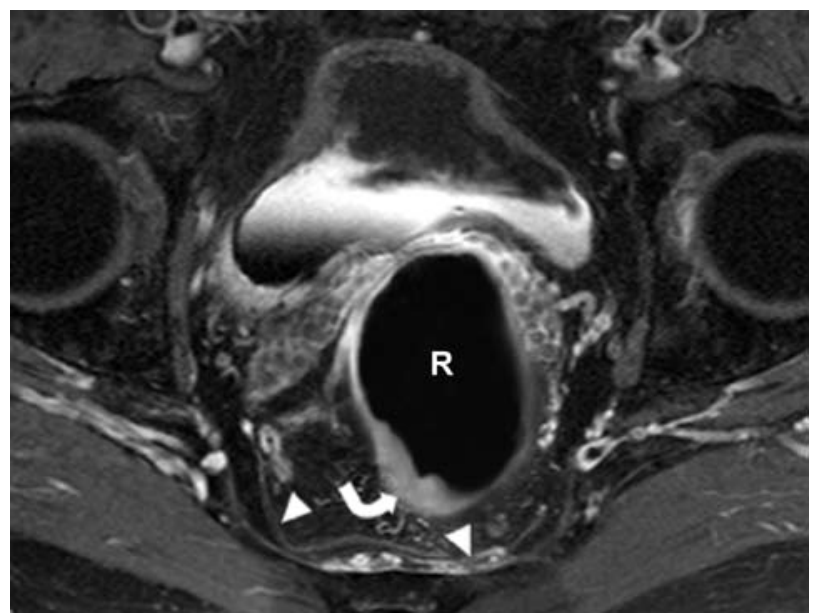

Figura 2b. El mismo caso 2 a, donde el tumor aparece limitado a la muscular (flecha curva) y la FMR, o plano quirúrgico, delimitando el mesorrecto.

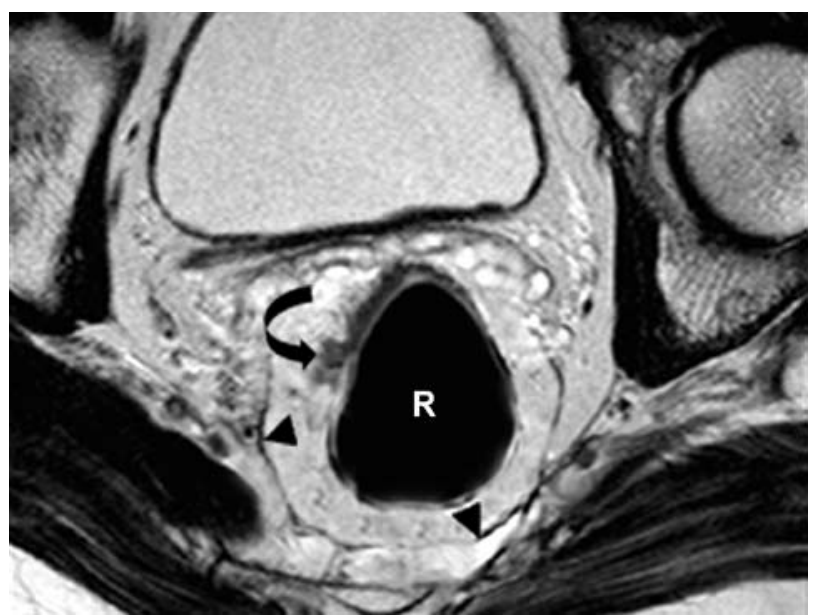

Figura 3a. Tumor en estadio T3 que sobrepasa el límite de la muscular (flecha curva) e invade la grasa del mesorrecto, sin alcanzar la FMR (cabezas de flechas). 


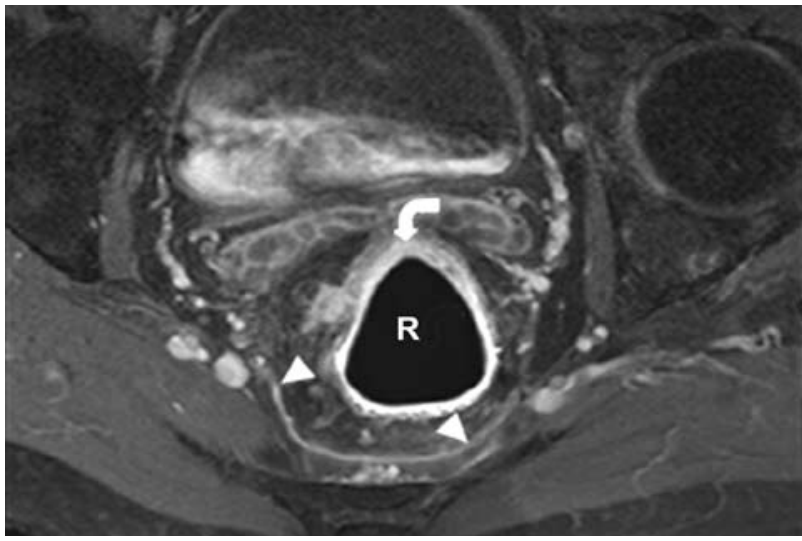

Figura 3b. Tumor y capa muscular aparecen hiperintensos y resaltan marcadamente, dada la supresión de la señal de la grasa del mesorrecto. La FMR (cabezas de flechas) se hace muy evidente.

En este sentido, en los tumores en etapa T3 es importante definir el límite del mesorrecto dado por la FMR, y la distancia del tumor o adenopatías a la FMR. Esta distancia es el marcador más preciso de predicción de recurrencia tras la cirugía, ya que estadísticamente el $15-20 \%$ de las cirugías con técnica de TME tiene margen positivo, por lo que el riesgo de recurrencia es inversamente proporcional a la distancia del tumor a la FMR. Por esta razón, en este estadio el tratamiento generalmente es quirúrgico y se asocia a un tratamiento coadyuvante previo a la cirugía con el fin de disminuir este riesgo de recurrencia ${ }^{(8)}$. De igual forma, aquellos pacientes en que se determina un margen MRC menor a $5 \mathrm{~mm}$ se benefician de quimio y radioterapia previas a la cirugía ${ }^{(7)}$.

Los cánceres rectales en estadio T4, es decir, aquellos que comprometen estructuras vecinas tales como vísceras, músculos, etc, en general no presentan dificultad diagnóstica a la RM (Figura 4). Sin embargo, debe tenerse presente que aquellos tumores T3 que comprometen la FMR, para efectos prácticos deben ser considerados como T4.

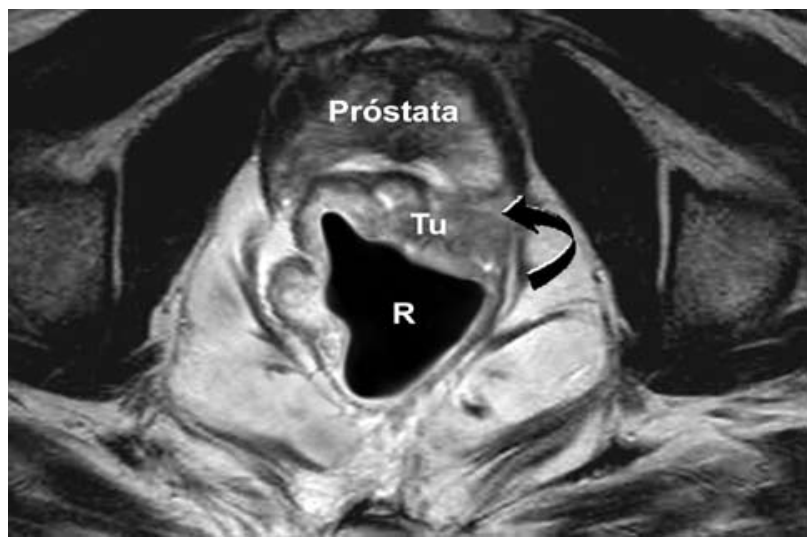

Figura 4. T2 axial. Tumor (Tu) invade las vesículas seminales y la próstata.
La diseminación ganglionar del cáncer rectal se desarrolla a través de dos rutas: cadena mesentérica inferior y cadena ilíaca interna, dependiendo si se trata de compromiso del recto superior e inferior respectivamente. La valoración por RM del compromiso linfático se basa en criterios morfológicos tales como tamaño, forma y contorno de los ganglios, lo que parece insuficiente tomando en consideración los siguientes hechos:

a. Diversos estudios han demostrado que el cáncer rectal frecuentemente desarrolla micrometástasis ganglionares y que, en más de la mitad de los casos los ganglios metastáticos miden menos de $5 \mathrm{~mm}$. y se encuentran a menos de $3 \mathrm{cms}$ del tumor ${ }^{(9)}$.

b. Es imposible distinguir entre ganglios aumentados de tamaño en forma reactiva, de aquéllos metastásicos.

Se encuentra en desarrollo el uso de contrastes específicos, basados en la captación de micropartículas de hierro por el sistema reticuloendotelial normal, para ser utilizados como predictores del compromiso ganglionar empleando criterios como la irregularidad de los bordes, la intensidad de señal y heterogeneidad de la señal, sumados a la espiculación de los bordes ganglionares ${ }^{(10,11)}$. Los ganglios normales experimentan una caída en la intensidad de señal en T2, no así los ganglios metastásicos ${ }^{(12,13)}$.

En resumen, en la actualidad el rol de la RM en la evaluación ganglionar, específicamente de los ganglios del mesorrecto, está basado en precisar si existen o no ganglios, su tamaño, su número (que permite definir el "N" de la clasificación TNM), la ubicación de éstos fuera o dentro del mesorrecto $y$, en el caso de estos últimos, su distancia de la FMR. Si bien la literatura asume como patológico un linfonodo mayor de $5 \mathrm{~mm}$, en nuestra práctica preferimos usar $3 \mathrm{~mm}$ como límite (Figura 5a, b).

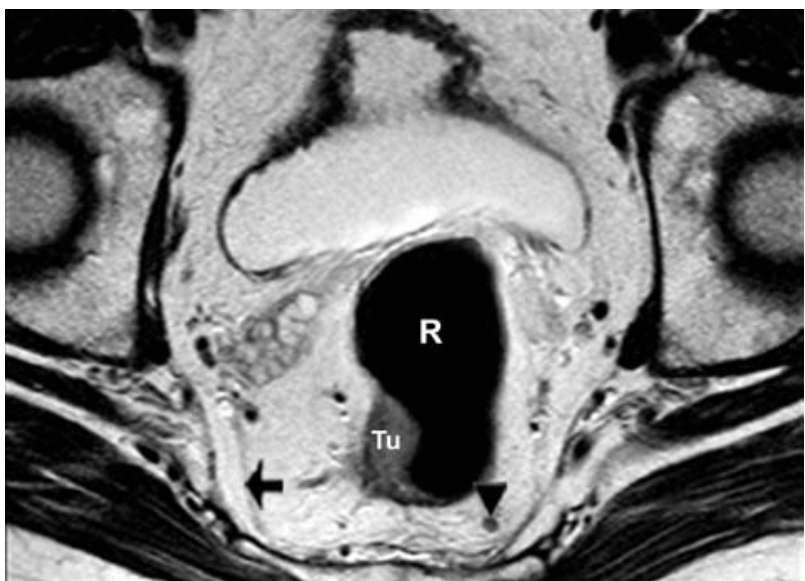

Figura 5a. Tumor (Tu) en estadio T3: compromiso de la grasa perirrectal con un linfonodo menor de $5 \mathrm{~mm}$ (cabeza de flecha) claramente identificado por su baja señal en comparación a la grasa vecina que es hiperintensa. La FMR se identifica claramente (flecha). 


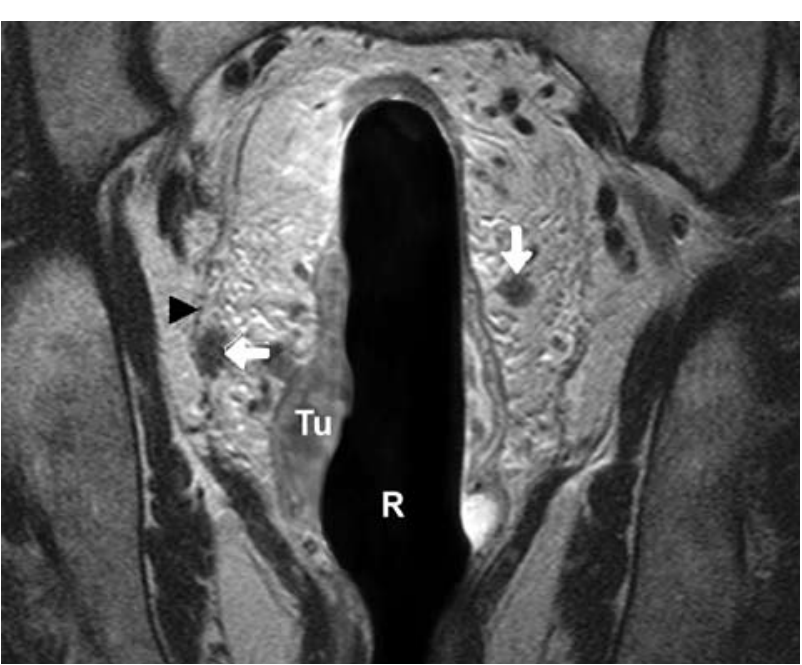

Figura 5b. Tumor en etapa T3, con ganglios de aproximadamente $1 \mathrm{~cm}$ de diámetro (flechas), uno de los cuales, a derecha, se ubica en contacto con la FMR (cabeza de flecha).

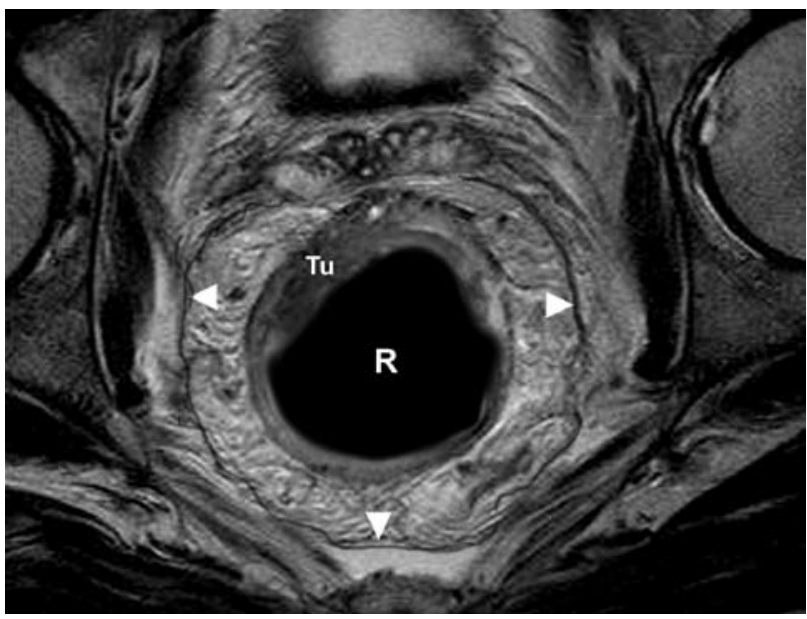

Figura 6. Marcado compromiso del mesorrecto, secundario a tumor rectal. No es posible diferenciar entre verdadero compromiso neoplásico o reacción desmoplástica; ambas situaciones son similares desde el punto de vista de la RM.

La técnica quirúrgica en el cáncer de recto es diferente a la cirugía del cáncer del resto del colon, lo cual está determinado por la anatomía de la zona y por el grado de extensión de la enfermedad en relación a las estructuras anatómicas locales. La mucosectomía está indicada en aquellos cánceres superficiales, mientras que cuando existe compromiso transmural (T2 y T3) la técnica estandarizada es la RTM(2). El plano de sección está dado por la FMR; cuando existe compromiso de la FMR o invasión de órganos vecinos (T4), es mandatario la radioterapia y/o quimioterapia previa para lograr una reducción tumoral y con ello mejor resultado con la cirugía.

\section{Conclusiones}

El tratamiento del cáncer rectal es quirúrgico y los pilares fundamentales del abordaje están orientados a: a) control de recidiva local, b) sobrevida a largo plazo, c) preservación del esfínter anal, vejiga y función sexual y d) conservación de una adecuada calidad de vida.

La introducción de radio y quimioterapia preoperatoria y la variedad de abordajes quirúrgicos disponibles requiere que las distintas técnicas imaginológicas entreguen una adecuada y segura etapificación del cáncer rectal con el objeto de seleccionar adecuadamente aquellos pacientes con mayor riesgo de recidiva local, para poder definir el tratamiento coadyuvante prequirúrgico y el abordaje quirúrgico propiamente tal.

El grado de diseminación local comprende la evaluación del compromiso mesorrectal y la determinación del MRC así como también del compromiso de estructuras pelvianas vecinas, incluyendo los ganglios del mesorrecto y de las cadenas ilíacas.

En el cáncer superficial, que es tratado sólo con resección quirúrgica local, la endosonografía y la RM con bobina endorrectal son los métodos más seguros en la valoración del compromiso mural. La endosonografía es un método seguro en la valoración del compromiso mural. Las principales desventajas de este método radican en ser operador dependiente y en su bajo rendimiento en la valoración del compromiso mesorrectal. La RM es similar a la endosonografía en su capacidad de evaluar las lesiones superficiales pero es superior en la evaluación del mesorrecto incluyendo los ganglios, FMR y estructuras vecinas. La RM es también superior a la TC en la evaluación del compromiso secundario de estructuras pelvianas.

El compromiso ganglionar sigue siendo un problema diagnóstico, si se basa sólo en criterios morfológicos. Investigaciones recientes en relación al uso de contraste paramagnético como el óxido de hierro y la valoración ganglionar en base a la homogeneidad de su intensidad de señal prometen ser un considerable aporte en este sentido. Distintos estudios demuestran que la seguridad de la RM en la etapificación TN varía entre $86-100 \%{ }^{(14,15)}$.

En conclusión, en la actualidad la adecuada etapificación y planificación quirúrgica del cáncer de recto lograda con los estudios imaginológicos junto a la estandarización de la técnica quirúrgica (RTM) sumados al uso de radio y quimioterapia previa a la cirugía, han permitido reducir su mortalidad a cifras cercanas a un $2,4 \%$ a dos años ${ }^{(8)}$.

\section{Bibliografía}

1. Levi F, Lucchini F, Negri E, La Vecchia C. Trends in mortality from Major Cancers in The European Union, including Acceding Countries, in 2004. Cancer 2004; 101: 2843-50.

2. Instituto Nacional de Estadísticas. Anuario de De- 
mografía y Estadísticas Vitales de Chile, 1990-2003.

3.. Heald RJ, Ryall RD. Recurrence and survival after total mesorectal excision for rectal cancer. Lancet 1986; 1 : 1479-1482.

3. Kapiteijn E, Marijnen CA, Nagtegaal ID, et al. Preoperative radiotherapy combined with total mesorectal excision for resectable rectal cancer. $\mathrm{N}$ Engl J Med 2001; 345: 638-646.

4. Quirke P, Durdey P, Dixon MF, Williams NS. Local recurrence of rectal adenocarcinoma due to inadequate surgical resection: histopathological study of lateral tumor spread and surgical excision. Lancet 1986; 2: 996-999.

5. Brown G, Richards CJ, Newcombe RG, et al. Rectal carcinoma: thin-section MR. imaging for staging in 28 patients. Radiology 1999; 211: 215-222.

6. Maldjian C, Smith R, Kilger A, Schnall M, Ginsberg G, Kochman M. Endorectal surface coil MR imaging as a staging technique for rectal carcinoma: a comparison study to rectal endosonography. Abdom Imaging 2000; 25: 75-80.

7. Beets-Tan RG, Beets GL, Vliegen RF, et al. Accuracy of magnetic resonance imaging in prediction of tumorfree resection margin in rectal cancer surgery. Lancet 2001; 357: 497-504.

8. Kapiteijn E, Marijnen CA, Nagtegaal ID, et al. Preoperative radiotherapy combined with total mesorectal excision for resectable rectal cancer. N Engl J Med
2001; 345: 638-646.

9. Monig SP, Baldus SE, Zirbes TK, et al. Lymph node size and metastatic infiltration in colon cancer. Ann Surg Oncol 1999; 6: 579-581.

10. Kim JH, Beets GL, Kim MJ, Kessels AG, Beets-Tan RG. High resolution MR imaging for nodal staging in rectal cancer: are there any criteria in addition to the size? Eur J Radiol 2004; 52: 78-83.

11. Brown G, Richards CJ, Bourne MW, et al. Morphologic predictors of lymph node status in rectal cancer with use of high-spatial-resolution MR imaging with histopathologic comparison. Radiology 2003; 227(2): 371-377.

12. Bellin MF, Roy C, Kinkel K, et al. Lymph node metastases: safety and effectiveness of MR imaging with ultrasmall superparamagnetic iron oxide particles-initial clinical experience. Radiology 1998; 207 :799-808.

13. Bellin MF, Beigelman C, Precetti-Morel S. Iron oxideenhanced MR lymphography: initial experience. Eur J Radiol 2000; 34: 257-264.

14. Beets-Tan RG, Beets GL. Rectal cancer: review with emphasis on MR imaging. Radiology 2004; 232: 335346.

15. Blomqvist L, Machado M, Rubio C, et al. Rectal tumor staging: MR imaging using pelvic phased-array and endorectal coils vs endoscopic ultrasonography. Eur Radiol 2000; 10: 653-660. 